IOS Press

\title{
Mediating individual affective experience through the emotional photo frame
}

\author{
Dong Keun Kim ${ }^{\mathrm{a}}$, Yongjoo Cho ${ }^{\mathrm{a}}$ and Kyoung Shin Park ${ }^{\mathrm{b}, *}$ \\ ${ }^{a}$ Department of Media Software, Sangmyung University, 7 Hongji-dong, Jongno-gu, Seoul, 110-743, Korea \\ E-mail: \{dkim,ycho\}@smu.ac.kr \\ ${ }^{\mathrm{b}}$ Department of Applied Computer Engineering, Dankook University, 152 Jukjeon-ro, Suji-gu, Gyeonggi-do, \\ 448-701, Korea
}

\begin{abstract}
A photograph is considered a medium with emotional legibility and a means of expressing and exchanging emotional experience. This research presents the interactive emotional photo frame system focusing on mediating individual affective experience among close relationships. This emotional photo frame system dynamically controls the photograph based on a user's affective states and user-specified emotion reaction rules. It is designed for helping users recognize and experience the same emotions with others. The system consists of an emotion recognition system, an emotion share server and an emotional digital picture frame. The emotion recognition system analyzes photoplethysmography, skin temperature and galvanic skin response signals to recognize user's emotional states and transmits the results to the server in real-time. The server stores user emotional data with individual emotion reaction rules that define how the picture frame should affect the photo in response to individual affective states. The emotional picture frame renders personalized visual and aural elements on the photograph according to the feedback of emotions. This paper presents an empirical exploration of the effectiveness of this system. The results revealed that most participants were influenced by their partner's emotion presented in this system. There was a strong relation of emotion sharing between the partners.
\end{abstract}

Keywords: Mediating, affective experiences, physiological signals, emotional expression, digital photography

\section{Introduction}

Photography can help people record and document family moments, capture phenomena and express the professional artistic images. With the advent of digital media, photography has become increasingly popular in daily life, with nearly sixty million photos being taken per day [34]. Such popularity has driven the creation of various kinds of devices and applications, such as digital camera, digital photo printers, digital picture frames, camera phones, photo-editing software, and online photo sharing services. Digital photography in conjunction with the Internet has enabled people to share their photos with friends and family members, supporting social interaction and affective awareness [19] and building social networks as

*Corresponding author. E-mail: kpark@dankook.ac.kr. in Flickr and Facebook. Especially, photo sharing has allowed people to share experience and emotion with others through pictures. Emotion often involves a social process in which a user communicates with others by creating emotional experiences [8]. There have been many previous works that used an interactive artifact for conveying personal emotion to others [11,27]. However, the way emotions are understood must either be pre-determined or agreed upon by users. For example, hapticons for enriching text messages on the instant messaging system are vibration patterns with a predefined emotional meaning (such as big smile and embarrassed) [27]. BuddyBeads support the expression of user emotions through private agreed codes [11]. Even though users share the same affective states, their expressions may be different. Hence, the uniform responses in emotional contents make it difficult for users to adequately express their emotions [33]. 


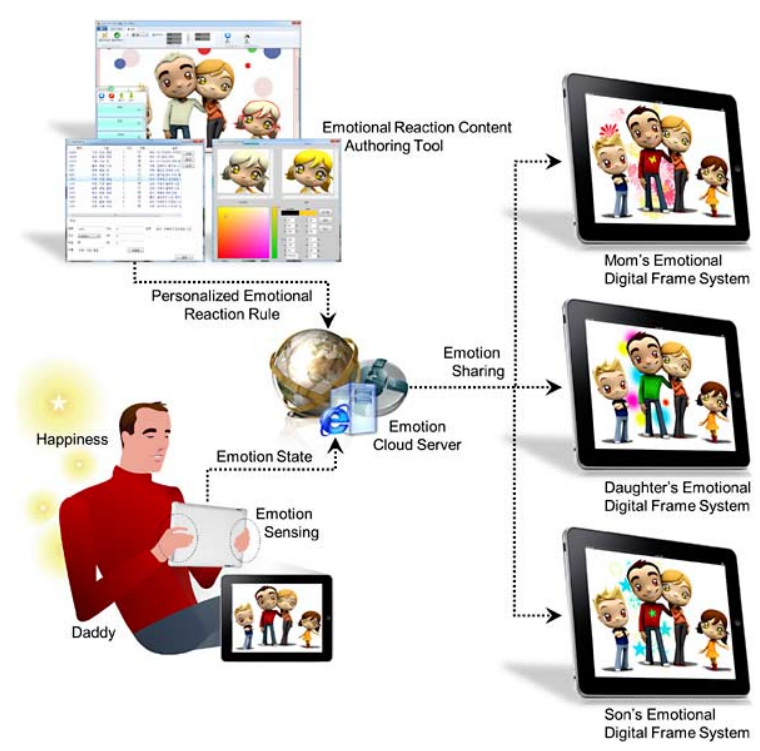

Fig. 1. A scenario of sharing individual affective experiences among family members through the interactive emotional digital photo frame system.

This research proposes the interactive emotional digital photo frame system designed for mediating user's affective experience among intimate relationships such as family members and couples. This system captures user's affective states passively by inferring from physiological signals. It dynamically adjusts visual and aural rendering of the photograph in correspondence with individual emotional changes. The emotional expression displayed on the photo reflects personal color preferences and visual effect patterns specified in the picture frame. This system facilitates the emotional photographs shared automatically via the server without requiring users to actively input for emotion sharing.

Figure 1 shows a scenario of family living in distance but sharing their emotions through this proposed system. When Dad touches the emotional digital photo frame, his affective state is read by the physiological sensors on the frame. Then the state gets propagated to his remote family members. Consequently, the remote family members can witness changes in Dad's emotions through the changes of the photo on their picture frame as the content changes its appearance in realtime. The visual and aural elements of the photo, such as saturation, luminosity, brightness, background audio and sound effect, change to reflect the user's current affective states. Each family member can design his or her own visual and aural elements on the photo with his or her preferred emotional expressions.
Recently as more increased numbers of people are in long-distance relationships, it is necessary to find more effective means of communication for emotional expression over a distance. In contrast to previous work, this emotional photo frame system encourages remotely located people to reciprocate their affective states in real-time. The primary goal of this research is to develop the system that mediates one user's affective states to induce a similar emotion in the other. This system is focused on facilitating affection transfer between distant users by capturing one's emotion non-intrusively using ANS physiological sensors and rendering them as personalized emotional expression that remote users can intuitively understand.

This paper is organized as follows. Section 2 describes a review of the literature on the emotion sharing picture system. Section 3 provides a brief overview on emotion model and emotion recognition using physiological signals of the Autonomic Nervous System (ANS) responses. Section 4 discusses the design and implementation of the proposed interactive emotional digital photo frame system prototype. Sections 5 and 6 describe the experimental methods, results and discussions. Finally, the conclusions and future directions are summarized in Section 7.

\section{Related work}

There have been many previous works that used a picture frame as a way to convey personal emotion to others. LumiTouch developed by MIT Media Lab is a pair of interactive photo frames designed for enhancing emotional communication between loved ones [2]. The photo frame consists of light-emitting elements and pressure sensors to detect if users took hold of the frame with their hands. It then displays different color lights based on how long and where users hold the frame. This frame also shows ambient light glows to indicate the presence of the other user if the other user is in front of the frame. These features enable to display user emotions and develop an abstract emotion language for basic communication.

Second Life is an online virtual world where users are represented as avatars and they can meet, exchange information and interact with each other. It is used to foster business and personal relationships. The EmoHeart project developed by University of Tokyo took Second Life one step further by actively analyzing user text inputs to discover user emotions and then displaying user emotional states on the avatar's face or as a heart on the avatar's chest [20]. 
In the Emoti-Picture frame system, a tangible interface has been added to the digital photo frame for emotion sharing with remote users [21]. This system consists of the picture area (on the left) and the feeling area (on the right). The picture area shows the photograph of the remote user's emotions. The feeling area consists of emotion buttons and heart-shaped emotion indicator used for expressing user emotion. The emotion buttons are used to express user emotion on the photo to the remote user. The related photo is displayed on the frame along with the heart-shaped indicator blinking.

Yamanishi's work [35] and EmuPlayer [22] are the music selection and recommendation system based on user emotion. The former work presented an emotional song selection system based on the acoustic-emotion model, which relates the acoustic fluctuations of a music concerning time variation of pitch, volume, and rhythm, using subjective evaluation. This system allows a user to present an emotional adjective and the degree of the emotional state. EmuPlayer recognizes user's emotion using skin temperature and heart rate data. Then, it generates a playing list of music based on the user's emotional state. This system was evaluated to measure the song's effect on a user's emotion before and after listening to the music, not to give the user bad influence.

Kim et al. designs and implements the early prototype of the Emotionally Intelligent Content framework [16]. Its primary focus was to provide the capability of sharing an emotional avatar within contents. The emotional avatar presented a user's current emotional status with different emotion color model. The system also worked in a simple direct communication mode between the emotion recognition and emotion rendering system. A virtual reality and an emotional game on Apple iOS platform were constructed using the prototype framework.

Whang et al. implements a two-dimensional emotion rule base system using physiological sensors to find valid physiological parameters and maps them using nine categories of human emotions [37]. They adopted a two-dimensional space made up of valence and arousal where emotions were classified in each of the four quadrants using two bipolar axes: pleasantness (P) vs. un-pleasantness (U) and arousal (A) vs. relaxation $(\mathrm{R})$ [18]. This research also developed a specially designed measurement device called "emotional mouse" for the recognition of EDA (Electro Dermal Activity), SKT (Skin Temperature), and PPG (PhotoPlethysmography). This research mainly discussed the rule base for determining a user's emotion based on the values measured using these physiological sensors.

While these works have explored various techniques to enhance interpersonal relationships, most of these cases require the user to actively express emotions. Furthermore, the way emotional expressions are understood has either been pre-defined or a new language must be established that both users have agreed to. The platform described in this research enables the mediation of user affective experiences using emotion recognition based on physiological signals where emotions can be shared without any work on the part of the user. This system also provides personalized emotional expression for users to intuitively understand affective states of the others with no additional learning or agreement needed for emotional expression.

\section{Emotion model and emotion recognition}

\subsection{Emotion response model}

Emotion classification is challenging for both humans and machines due to a large variety of emotional states. In previous research, psychologists distinguished two emotion models: discrete emotion categories and two or three continuous dimensional emotional models [32]. The discrete emotion categories originated from Darwin's pioneering work on basic emotion. It includes six basic emotions: anger, disgust, fear, joy, sadness, and surprise [15].

On the other hand, the bi-dimensionality theory of emotion proposes that the nature of emotional experience is primarily determined by two main dimensions, namely, valence and arousal. In this theory, valence and arousal are two orthogonal, independent dimensions of the emotional stimulus [7]. The dimensional model allows one to map discrete emotion labels onto a two dimensional coordinate system. The affective dimensions are continuous, and so are interesting for real-time monitoring of user emotion. Moreover, this dimensional model of representation allows for the computation of changes in user emotional state, which is needed to represent emotion as a dynamic process [14].

This research adopted Russell's emotion model, where the two dimensions are represented by a vertical arousal axis and a horizontal valence axis [28]. Figure 2 shows the emotion response model representing the five emotional categories: Neutral, Pleasure, Stress, Fatigue, and Relaxing. This two-dimensional extended 


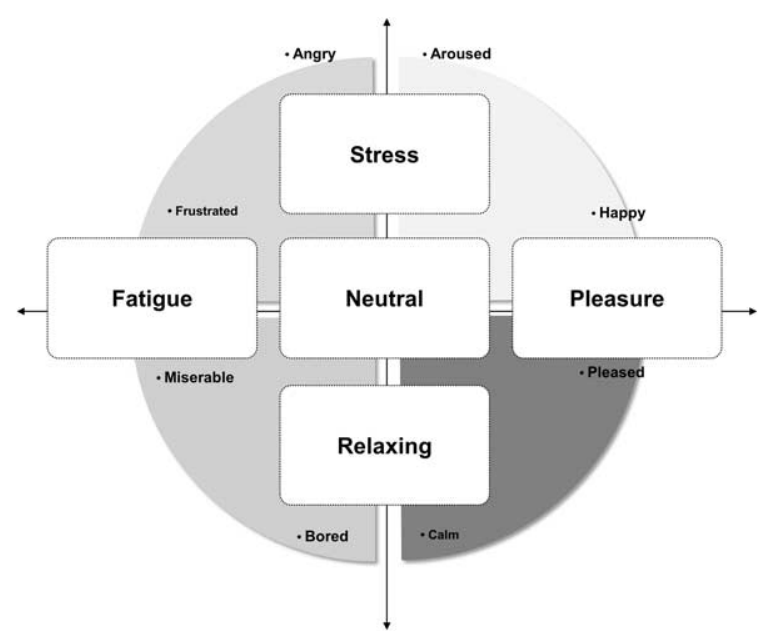

Fig. 2. Emotion response model representing the five emotional categories: Neutral, Pleasure, Stress, Fatigue, and Relaxing.

emotion model is used to represent the user's current emotional state in the five different categories based on continuous physiological changes and the measurement of affective states.

\subsection{Emotion recognition using ANS physiological signals}

A great deal of research has already been conducted on emotion detection through non-verbal communication methods, such as physiological signals and expressive responses [3,5,24,36,39]. Retrieving emotional states using expressive responses (such as facial, vocal and postural expressions) requires computer vision, speech or gesture recognition technology. Identifying user emotions based on these techniques can lead to the development of flexible user interfaces. However, it requires sophisticated data pre-processing steps, such as image region, motion detection and lexical analysis that involve machine learning techniques for feature extraction. Also, this technique is easily affected by some environmental factors, such as lights, colors and noise.

Emotion recognition using physiological signals (such as blood pressure, skin temperature, pupillary response, brain waves and heart response) have provided promising results in cases where the affective states are directly related to changes in bodily signals $[9,26,30]$. Recently, much work has been devoted to using CNS (Central Nervous System) responses, such as EEG signals, for the classification of emotion states using both artificial and statistical methods. However, it is a challenging issue to extract the fea- tures to achieve optimal classification of emotions as described in Eum et al. [6] and Park et al. [23].

On the other hand, ANS (Autonomic Nervous System) responses can be processed in real-time to capture indirect perceptual emotional responses [10,17]. ECG (Electrocardiogram) signals provide more precise information about HRV (Heart Rate Variability) features [31]. HRV is frequently used to analyze time and frequency and the response of the autonomic nervous system. However, ECG requires the attaching of electrodes to the user's chest. Furthermore, ANS activities are involuntary responses, so they cannot be controlled and are referred to as "fake emotions" [14].

Changes in the amplitudes of PPG signals are related to the levels of tension in human [4,15]. Anger induces a large increase in skin temperature, whereas fear and sadness induce lower variations. Major magnitude changes in GSR signals are related to emotional excitement and dynamic activity in humans $[4,37]$. The most important reasons for using ANS physiological signals are that physiological signals based on ANS activity are very descriptive and easy to measure. Moreover, it is possible to obtain reliable representations of true emotion that cannot be actively controlled [12].

According to previous study associated with these physiological parameters [1,9], the feasibility of emotion classification was highly accurate up to $80 \%$ and 99\%, using the Support Vector Machine (SVM) algorithm. Although these results are promising, these parameters can frequently differ from one user to another and these features are very sensitive to different algorithms. They also require sophisticated data prepreprocessing. Therefore, this proposed emotional digital photo frame system estimates the affective states of individual users by adopting the emotion rule base which is based on heuristic emotion assessments extracted from ANS-based signals, such as PPG, SKT, and GSR responses. This method is significantly acceptable up to $71.67 \%$ for identifying emotional responses, as it has been previously reported $[1,12,13$, $15]$.

\section{System implementation}

It is assumed that photographs are effective contents for evoking emotional experiences between close friends and family members. However, each individual has a unique personality and may feel differently while viewing the same photograph. It has been shown that people also preferred user interactions so that they were able to directly control the contents by 


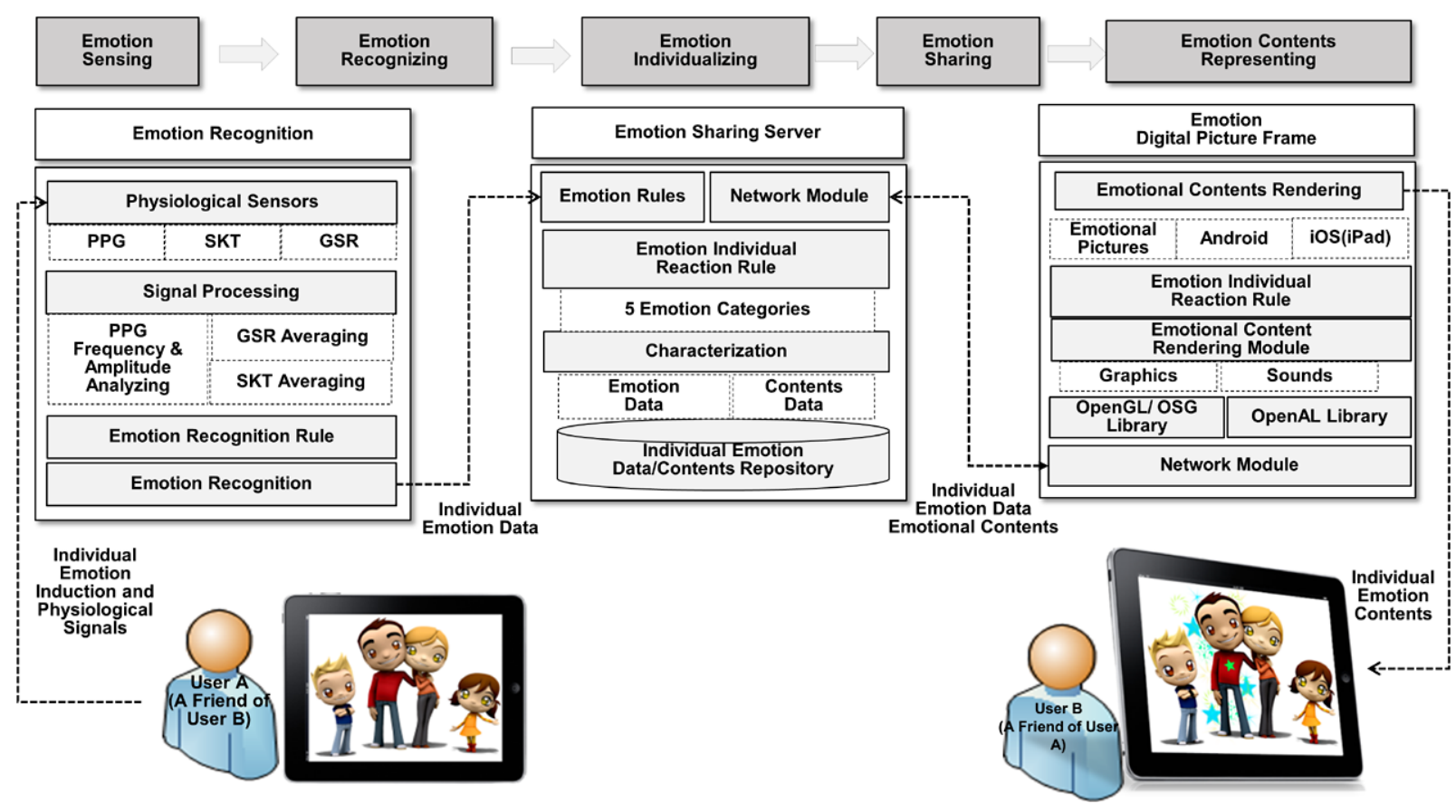

Fig. 3. The emotion sharing process between two remote users through the interactive emotional digital photo frame system.

themselves [29]. This system allows the individualized emotional rules to be shared automatically, once the rules are put into place. Then, the rules determine how the emotional photograph will be rendered to reflect their personal preference.

The interactive emotional digital photo frame system is built on top of the Emotionally Intelligent Contents framework [25]. This system is composed of three subsystems: (1) an emotion recognition system based on individual physiological data, (2) an emotion share server for sharing individual emotion data and contents; and (3) an emotional digital picture frame that renders emotional contents. The emotion recognition system receives a user's physiological signals and determines user's affective state by applying the emotion deduction algorithm on the signals. The emotion share server provides a personalized emotion-sharing service based on user's affective state along with emotion reaction rules and emotional content elements. The emotional digital picture frame renders emotional state changes of remote users through the emotion share server.

As shown in Fig. 3, the proposed system is comprised of the following representative operation processes: emotion sensing, recognizing, individualizing, sharing and representing for individual emotion data and contents. The system workflow and possible sce- narios for sharing individualized emotions are described as follows:

(1) When the local user, A, views the photograph while touching the physiological sensors on the frame, the emotion recognition system captures his or her physiological signals and identifies his or her current affective state.

(2) This affective state is then sent to the persistent storage on the emotion share server. In addition, the emotion share server stores the emotion reaction rules and emotion content elements for each individual.

(3) User B's emotional picture is rendered by the commands generated using User A's current emotional states, User B's emotion reaction rules and emotion content elements. The picture on the frame appears dynamically in real-time as new affective states are received from User A.

(4) The remote user, B, who can be a friend or family member of User A, may infer the emotional states of User A by changes appeared in the emotional photograph. As User B modifies his or her emotion rules or contents, the updates are automatically shared via the server to reflect on the photo. User A's affective states may induce a similar emotion in User B, which in turn feeds 


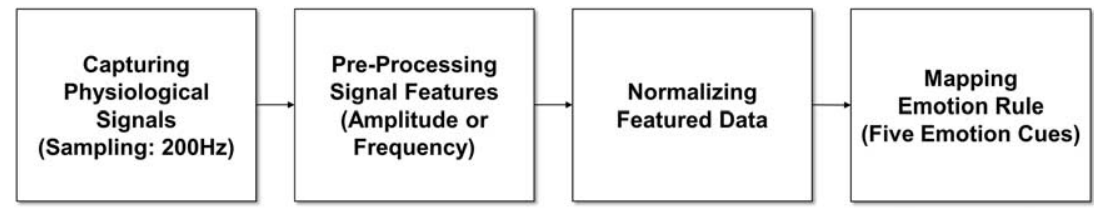

Fig. 4. A block diagram of emotion recognition procedure using PPG, SKT and GSR physiological signals.

into User A's matching emotions by sharing the interactive emotional digital photograph.

\subsection{Emotion recognition system}

The emotion recognition system estimates the user's affective states using ANS physiological sensor signals such as PPG (Photoplethysmography), SKT (Skin Temperature) and GSR (Galvanic Skin Response). PPG measures the cardiac synchronous changes in the blood volume. Changes in the amplitudes of PPG signals are related to the levels of tension [15,24]. SKT measures the thermal response of human skin. Anger induces a large increase in skin temperature, whereas fear and sadness induce lower variations. GSR measures the changes in the electrical resistance of the skin in response to autonomic arousal. Major changes in GSR are related to emotional excitement and dynamic activity [24,37].

Figure 4 shows the diagram of emotion recognition procedure. The analog-to-digital converter attached to this system generates packetized data using RSE (Referenced Single Ended) mode in real-time. In order to compensate for noises and artifacts, a pre-processing stage is performed, consisting of $0.5 \sim 3.0 \mathrm{~Hz}$ bandpass filters for signals, and for absolute measures, a DC filter is used including a $60 \mathrm{~Hz}$ notch filter for noise cancelling, respectively. The pre-processing PPG, SKT, and GSR data are extracted using a 200$\mathrm{Hz}$ sampling rate. Because of the use of three different signals, the emotion recognition system uses dynamic memory allocations with a circular queue memory structure to improve memory usage.

The PPG frequency and amplitude and the average values for the GSR and SKT signals are selected to extract features for emotion recognition every second. The mean amplitude is defined as the average value of the computed amplitudes [12]. For the real-time physiological signal processing, a sliding window technique and TDP (Time Dependent Parameter) was used, as referred in [12] and [15].

In order to minimize the individual differences in physiological signals, a normalization method is adopted as a reference, as studied in [13] and [19]. The normalization method is described by the following Eq. (1):

$$
E_{\mathrm{PPG}, \mathrm{GSR}, \mathrm{SKT}}=\frac{\left(C_{\mathrm{PPG}, \mathrm{GSR}, \mathrm{SKT}}-N_{\mathrm{PPG}, \mathrm{GSR}, \mathrm{SKT}}\right)}{N_{\mathrm{PPG}, \mathrm{GSR}, \mathrm{SKT}}}
$$

In Eq. (1), $E_{\mathrm{PPG}, \mathrm{GSR}, \mathrm{SKT}}$ denotes the percentage increase or decrease of the PPG, GSR, and SKT signals from the neural state ( $\left.N_{\mathrm{PPG}, \mathrm{GSR}, \mathrm{SKT}}\right)$ to the current state $\left(C_{\mathrm{PPG}, \mathrm{GSR}, \mathrm{SKT}}\right)$. The values of each signal data type are normalized by using the average value of the corresponding data collected during the emotion recognition phase for the same user.

In addition, the threshold band is designed as a neutral range. In Eq. (2), $E[x]$ denotes the mean values of the normalized PPG, SKT, and GSR data when $X_{k}$ represents each signal amplitude values regarding to the continuous time $(t)$. In Eq. (3), $S[x]$ denotes the standard deviation of the normalized PPG, SKT, and GSR data. In Eqs (4) and (5), the maximum and minimum range of the designed threshold, $T_{\operatorname{Max}}\left[X_{n}\right]$ and $T_{\text {Min }}\left[X_{n}\right]$, are calculated by a sliding window method. For example, when the size of the initial window is $10 s$, this contains 40 reference signal values, in which case $n=40$ in (4) and (5). The physiological signal is estimated every second by incoming signal data.

$$
\begin{aligned}
& E[x]=\lim _{N \rightarrow \infty} \frac{1}{N} \sum_{k=1}^{N} X_{k}(t) \\
& S[x]=\lim _{N \rightarrow \infty} \frac{1}{N} \sqrt{\sum_{k=1}^{N}\left(X_{k}(t)-E[x]\right)^{2}} \\
& T_{\operatorname{Max}}\left[X_{n}\right]=E\left[X_{n}\right]+S\left[X_{n}\right] \\
& T_{\operatorname{Min}}\left[X_{n}\right]=E\left[X_{n}\right]-S\left[X_{n}\right]
\end{aligned}
$$

Finally, the normalized physiological data are mapped to the regions of the heuristic two-dimensional emotion model adopted Russell's emotion model. 
Table 1

Emotion recognition rule base

\begin{tabular}{|c|c|c|c|c|}
\hline $\begin{array}{l}\text { Emotion } \\
\text { Status }\end{array}$ & $\begin{array}{l}\text { PPG } \\
\text { Freq. }\end{array}$ & $\begin{array}{c}\text { PPG } \\
\text { Amplitude }\end{array}$ & $\begin{array}{l}\text { GSR } \\
\text { Mean }\end{array}$ & SKT Mean \\
\hline \multirow{4}{*}{ Pleasure } & + & + & 0 & - \\
\hline & + & + & 0 & 0 \\
\hline & + & 0 & 0 & + \\
\hline & + & + & - & - \\
\hline \multirow{4}{*}{ Stress } & 0 & - & 0 & - \\
\hline & 0 & 0 & 0 & - \\
\hline & - & - & + & + \\
\hline & - & + & + & - \\
\hline \multirow{4}{*}{ Fatigue } & - & 0 & + & 0 \\
\hline & - & + & + & + \\
\hline & + & 0 & 0 & 0 \\
\hline & + & 0 & + & - \\
\hline \multirow{4}{*}{ Relaxing } & - & + & - & - \\
\hline & - & + & - & 0 \\
\hline & - & 0 & 0 & 0 \\
\hline & - & 0 & + & - \\
\hline \multirow{2}{*}{ Neutral } & - & - & - & - \\
\hline & 0 & 0 & 0 & 0 \\
\hline
\end{tabular}

The “+”, “-”, and "0" symbols stand for "increase", "decrease”, and "no fluctuation", respectively.

Then, the emotion deduction algorithm [10] differentiates a user's emotional state into five categories (i.e., Neutral, Pleasure, Stress, Fatigue and Relaxing) based on user's continuous physiological changes (i.e., increase, decrease and no fluctuation).

Table 1 shows the simulated emotion rule base that is used for inferring user's emotional states, coded as Pleasure, Stress, Fatigue, Relaxing and Neutral. The designed threshold band can classify the increase or decrease of physiological signal patterns. The threshold range of normalized physiological signal patterns occurs at the state of Condition I-3.

Conditions I:

1) "+" symbol: $X_{k}(t)>T_{\operatorname{Max}}\left[X_{n}\right]$

2) “_" symbol: $X_{k}(t)<T_{\operatorname{Min}}\left[X_{n}\right]$

3) "0" symbol: $T_{\operatorname{Min}}\left[X_{n}\right] \leqslant X_{k}(t) \leqslant T_{\operatorname{Max}}\left[X_{n}\right]$

When the physiological signal values are plotted as threshold range values, "+" and "-" denote an "increase" or "decrease" in the normalized physiological signal values, respectively. The " 0 " symbol stands for "no fluctuation"; in other words, the normalized physiological signal values are affiliated to the threshold range.

For example, all-zero values of sensor signals indicates "Neutral" state, which will be used as a refer- ence. When the values of the frequency and amplitude of PPG signals increase and there is no deviation in the mean values for GSR and SKT signals, this pattern is considered as the "Pleasure" emotional state.

\subsection{Emotion share server}

The emotion share server provides storage and retrieval services for each individual's accumulated emotion data (such as affective states, emotion reaction rules) and emotional content elements. The server stores the user's raw physiological data and affective states, retrieved by the emotional recognition system, in real-time. It also notifies the current user's affective state to all emotion digital picture frames that are connected to the server. The emotion share server is implemented using the REST (Representational State Transfer) web service model with Apache Web server and MySQL database on a Linux platform.

In order to support the individual's personalized emotional response service, a unique identification number is given to each user along with his or her emotion data at given dates and times. Similarly, the emotion reaction rule management database has also been created for each emotion digital picture frame to handle customized feature regulations. The default emotion reaction rule set is provided as a starting point for the user to define his or her customized emotion reaction rules on how color, light and sound should be manipulated in the event. For example, the default rule contains light green, midnight blue, light steel blue, orange red, light goldenrod green, black color masks for Neutral, Fatigue, Relaxing, Pleasure, Stress states, respectively, with no specified pattern until a user defines his or her own rule in this picture frame.

The individual's emotion reaction rules and emotional content elements are created by using the GUIbased emotion content authoring tool [25]. This visual scripting tool is written in C\# and Windows Presentation Foundation (WPF) library. It provides much of the same basic image editing functionalities, such as primitive shape, color, object selection, region and layer, for defining more complex emotion rule and content elements. A user can load a photograph into the authoring tool, select one or more arbitrary regions for each emotional state, and then set different colors, shapes or patterns. The rules will be applied or placed on specific region of the image for each emotional state.

Figure 5 shows the example of the sample XMLbased emotional rules. The rule works on an image called "floor.png." A couple of polygonal regions are 


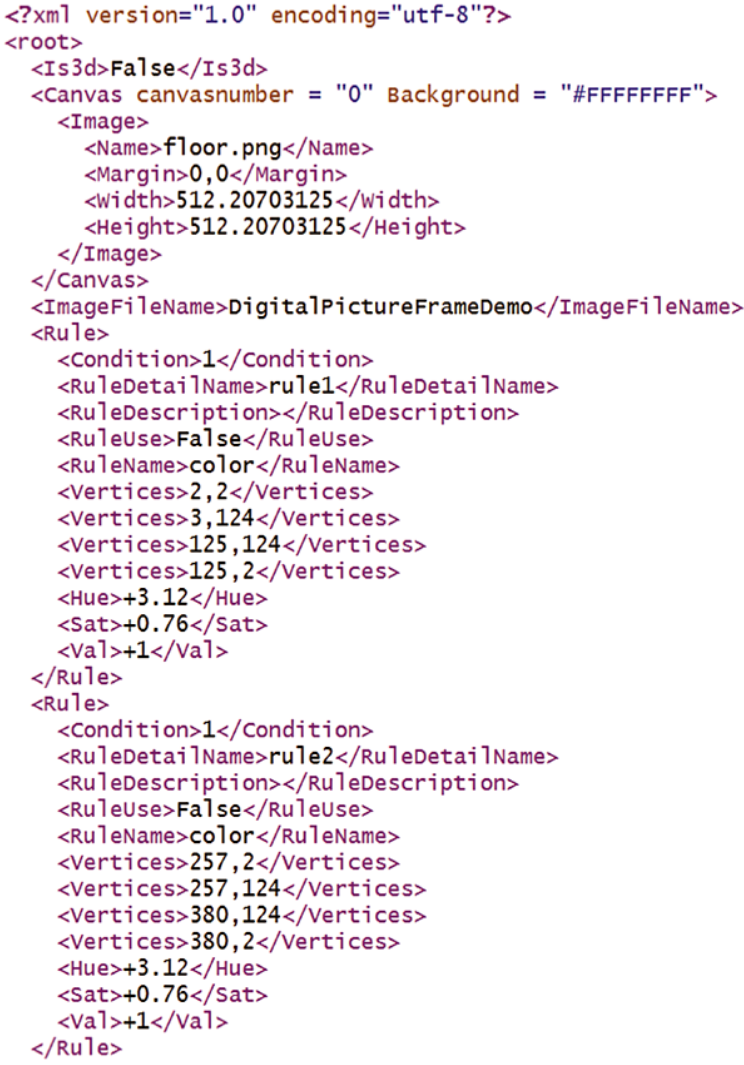

Fig. 5. An example of the emotional rules.

defined with several vertices. The HSV (Hue, Saturation, Value) color values are set for the regions specific to the "Pleasure" emotional state (i.e., 2). Unlimited number of regions can be created for each emotional state.

\subsection{Emotional digital picture frame}

The current prototype of emotional digital picture frame runs on Apple's iOS and Google's android tablets. The system is responsible for retrieving user's emotional data and processing them for emotion rendering on the photograph. It manipulates graphical maps by putting an emotional color and visual pattern mask on the original photo to create a new emotional content in real-time. Also, it dynamically changes its visual and aural aspects on the photo according to user emotion reaction rules specific to a given user's affective state.

When the system starts, it requests a user identification number so that it can keep track of the user. Then, it continuously interoperates with the emotion share server to obtain new emotion reaction rules and contents. The user emotion reaction rules are defined as a hierarchical structure of emotion rules containing condition, regions and actions for the photo [25]. They describe several certain actions (such as visual and aural effects) applied on some image areas (regions) for specific user affective states (conditions). The contents and the user emotion reaction rules are managed separately so that each user can specify his/her emotion rules without affecting other user's emotion rules.

The acquired emotional contents are rendered using the OpenGL ES 3D mobile platform graphics library and the OpenAL sound library. The various web-based communication features of the libcURL library were used for communication between the emotion picture frame and the emotion share server. In addition, open source libXML was used to handle XML for emotion reaction rules and content expression.

\section{Experiments}

The experiment was conducted to assess the system effectiveness and to the feasibility of mediating affective experiences between two users via the interactive emotional photo frame system. The participants were asked to identify their own affective states while viewing the emotional photo affected by their own emotion, followed by sharing with their partners' emotion.

\subsection{Participants}

In this study, fourteen undergraduate students (12 women and 2 men) were recruited and divided up into seven pairs. Each participant was paired with their close friend. The participants were 20 to 23 years of age with an average age of 21.5. All participants were majoring in computer science-related fields. They all had a moderate level of computer experience. Most of them had no previous experience with physiological sensors. None of them had previous experiences with an interactive emotional photo frame system. All participants expressed fairly high interest in playing with this system.

\subsection{Apparatus}

Figure 6 shows a snapshot of a subject using this system during the experiment. PPG signals were recorded with TSD200C sensors that were placed on the subject's earlobe and SKT signals were measured with TSD202A sensors placed on the subject's thumb. The subject wore these sensors while holding the dig- 


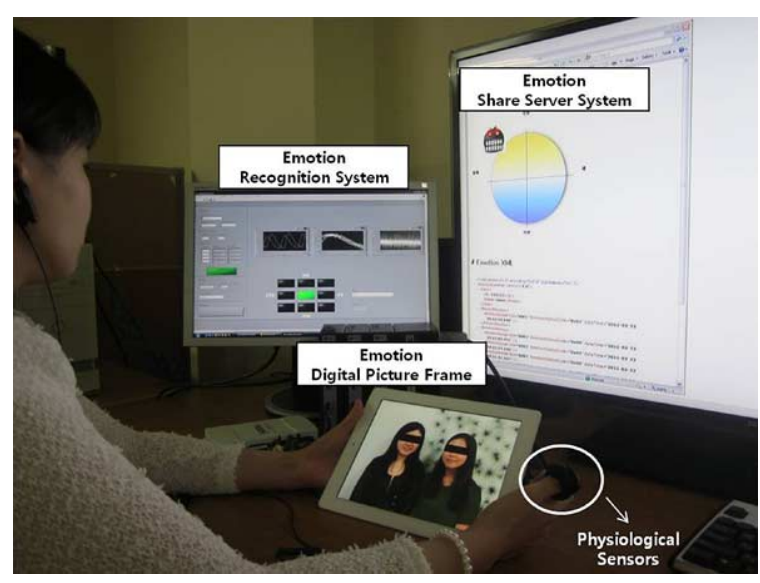

Fig. 6. A snapshot of a subject experiencing emotion transfer over the emotional digital picture frame with the emotion recognition and the emotion share server.

ital picture frame system. The GSR signals were measured with TSD203 using two electrodes which were attached to the index and middle fingers of the subject's right hand. All physiological data were sampled at $200 \mathrm{~Hz}$ using Biopac's MP100 system [12], and were analyzed with the emotion deduction algorithm written in LabView. The one-minute of data during the adaptation period was recorded for normalization. The emotional digital picture frame runs on Apple's iPad and Samsung's GalaxyTab.

The emotion reaction rule profile was pre-defined by each subject. The rules were then used to change the appearance of the clothes and background of the photograph around the subject or the subject's friend on the picture frame. Table 2 shows the example profile of the two subjects participated in the experiment. Both subjects chose different colors and visual patterns based on their preferences. For example, when Subject A's emotional state is "Pleasure", the personal preference cloth color is "HSV $\left(200^{\circ}, 74,93\right)$ " with the visual effect of blue hollow stars. When Subject B's emotional state is "Pleasure", the personal preference cloth color is "HSV $\left(56^{\circ}, 100,100\right)$ " with the visual effect of yellow solid stars. For example, "HSV (56 , $100,100)$ " represents yellow, which is the same color code as "RGB $(255,238,90)$ " and the color palate code of "\#FFEE00".

Figure 7(a) is the original photo with no effects shown in Subject A's digital picture frame. Figure 7(b) and (c) show the personalized effects and colors applied to the original photo according to Subject A's states of "Pleasure" and "Relaxing" on her picture frame, respectively. On the other hand, Fig. 7(d)
Table 2

Subject A and B specified color descriptions with visual patterns corresponding to their emotional states

\begin{tabular}{|c|c|c|c|}
\hline Subject & $\begin{array}{l}\text { Emotional } \\
\text { States }\end{array}$ & $\begin{array}{l}\text { Color Descriptions } \\
\text { (Emotion Content) }\end{array}$ & $\begin{array}{l}\text { Visual } \\
\text { Patterns }\end{array}$ \\
\hline \multirow{2}{*}{ Subject A } & Pleasure & $\operatorname{HSV}(200,74,93)$ & 命出會 \\
\hline & Stress & $\operatorname{HSV}(0,0,0)$ & $x * x$ \\
\hline \multirow{3}{*}{$\begin{array}{l}\text { (Friend of } \\
\text { subject B) }\end{array}$} & Fatigue & $\operatorname{HSV}(0,100,84)$ & 8,8 \\
\hline & Relaxing & $\operatorname{HSV}(337,91,96)$ & 情家眝 \\
\hline & Neutral & Transparent & None \\
\hline \multirow{2}{*}{ Subject B } & Pleasure & $\operatorname{HSV}(56,100,100)$ & औरी \\
\hline & Stress & $\operatorname{HSV}(124,18,28)$ & 豢䒹茟 \\
\hline \multirow{3}{*}{$\begin{array}{l}\text { (Friend of } \\
\text { subject A) }\end{array}$} & Fatigue & $\operatorname{HSV}(234,98,65)$ & 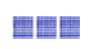 \\
\hline & Relaxing & HSV $(10,99,99)$ & 棠第靠 \\
\hline & Neutral & Transparent & None \\
\hline
\end{tabular}

demonstrates how Subject A's digital picture frame reflects Subject B's "Stress". This is because Subject A had previously defined that the visual effect of "black leaves" would depict the emotional state of "Stress". In other words, each digital picture frame follows the emotion rule to its owner even though the picture shows emotional states of the remote user. This mechanism allows each system owner to be aware of the other user's emotional states.

\subsection{Procedure}

The participants were first given a pre-test survey questioning technology and previous experiences with the emotion digital photo frame system and physiological sensors. Next, each participant was paired with a close friend and allowed to take pictures. Then, each participant created his or her own emotion reaction rule profile on the system. The participants performed the actual experiment by completing two tasks: (1) identifying their own affective states and (2) sharing the emotional experiences by their partner's affective states.

First, each subject performed the subjective emotion identification task to evaluate whether the subject's emotion measured using physiological signals matched his or her subjective feeling. When the subject watched the emotional photo, the visual appearance around the subject in the photograph (which was taken during the pre-test session) was dynamically changed by the subject's emotional state. Such an emotional (color or pattern) effect on the photo was based on the emotional profile specified by each subject during the pre-test session. 


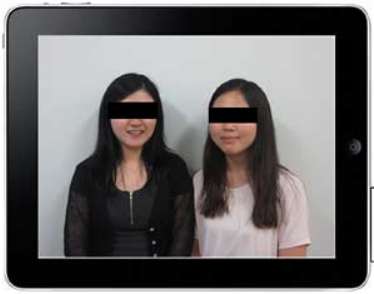

(a) Original Image-No Effects (Subject $A$ and $B$ )

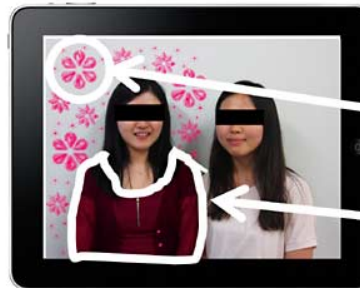

(c) Relaxing state of Subject $A$

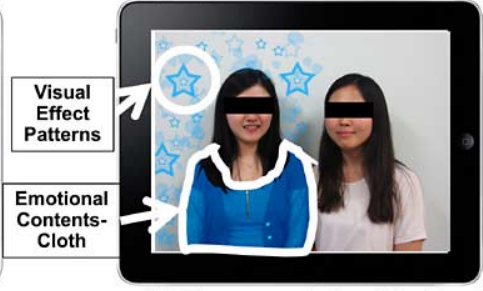

(b) Pleasure state of Subject A

(Cloth and Visual Effect with Blue Color)

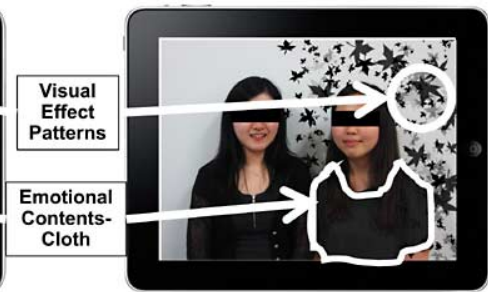

(d) Stress state of Subject B

(Cloth and Visual Effect with Pink Color)(Cloth and Visual Effect with Black Color)

Fig. 7. The color and visual effect changes in the picture according to subjects' emotion changes on Subject A's digital picture frame.

The subject's affective states recognized by the system were logged into a file. Also, the subject was asked about his or her subjective feeling at the moment whenever a transition in the subject's emotional state (for example, from "Neutral" to "Pleasure") was detected by the emotion recognition system. Then, the agreement between the emotional state detected by the subject's physiological signals (objective measurement) and the user-stated subjective feelings (subjective measurement) was measured while viewing the changes in the emotional photo.

Secondly, the subject performed the emotionsharing task to evaluate whether his or her emotions were influenced by his or her partner's emotions. The main objective was to measure how much the subject empathized with his or her friend via this system. Each subject was asked questions about his or her emotional feeling while looking at the emotional photo affected by his or her partner's emotions collected from the first task.

In this case, the color of the partner's clothes and the background visual effects around the partner in the photo were changed according to the rules specified by the subject's emotion profile. Then, the agreement how similar the subject's feelings matched the partner's affective states was analyzed to measure emotionsharing.

The experiment took about twenty minutes. For each task, three minutes were used for the relaxation (2-min) and adaptation (1-min) period, and the follow- ing two minutes were used for the actual task. A ten minute break was given to the participants between the two tasks.

\section{Results and discussion}

Figure 8 describes the patterns of Subjects A's physiological signals when she had experienced the changes of color patterns in the emotion picture frame under the "Fatigue (0x03)" affective state. Specifically, the frequency of PPG signals showed a decreased pattern ("-" symbol), and the amplitude of PPG signals and mean value of both GSR and SKT signals showed an increased pattern ("+" symbol).

For example, in Fig. 8 C. (a), the PPG frequency decreased from $1.5 \mathrm{~s}$ to $3.5 \mathrm{~s}$ so that it had the decreased symbol ("-"), whereas the amplitude of PPG signals and mean value of both GSR and SKT signals increased over the same period with the increased symbol ("+"), while watching the fatigue content was displayed in the emotional picture frame. It was found that the physiological patterns conformed to the emotion rules in this manner as described in Table 1.

\subsection{Agreement of individual emotion response}

Table 3 shows the results of the first task and the second task. First, it evaluated how much the emotional photographs reflect individual emotions by measuring the agreement of individual emotion response. The 


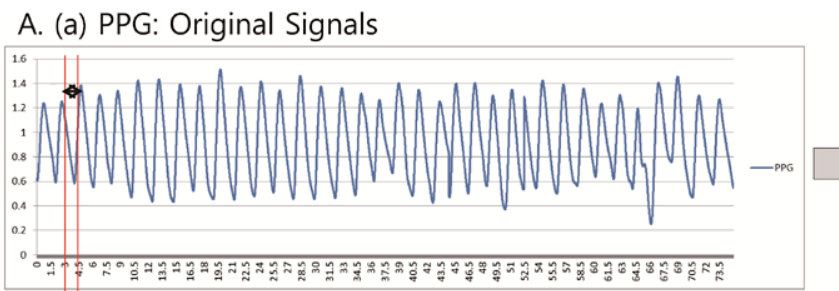

A. (b) GSR: Original Signals

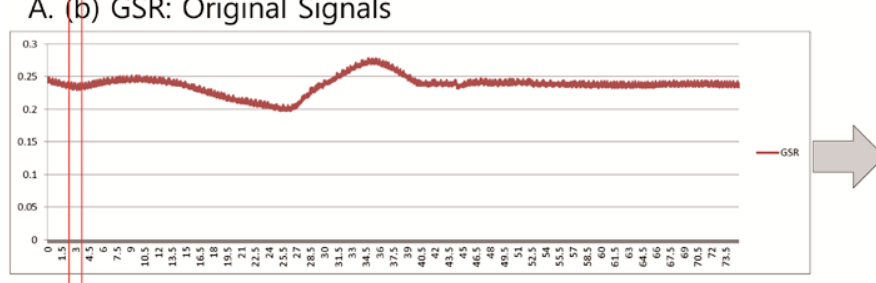

A. (c) SKT: Original Signals

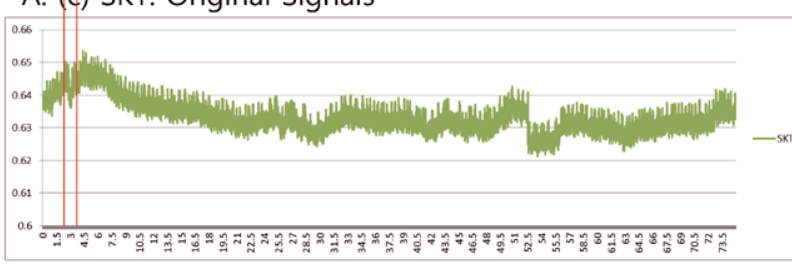

B. (a) PPG: Selected Signals (Magnified)

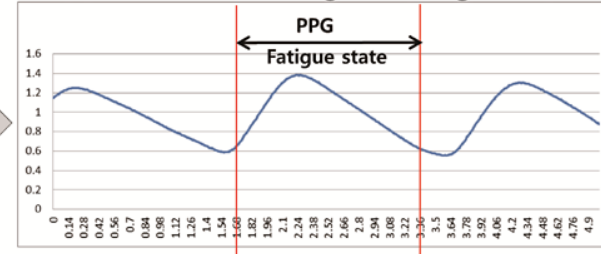

B. (b) GSR: Selected Signals (Magnified)

4.4.

ringminim

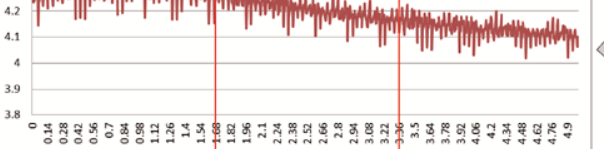

B. (c)-1 SKT: Selected Signals (Magnified)

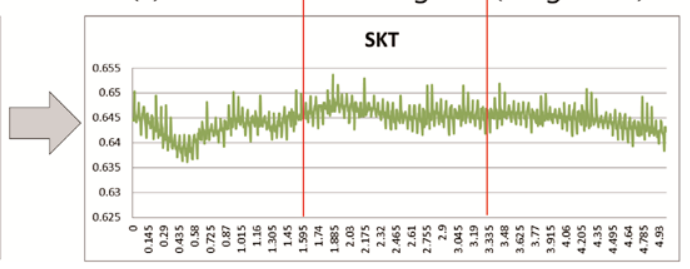

C. (a)-1 PPG: Preprocessed Signals-Frequency

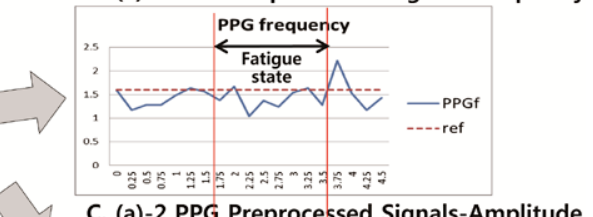

C. (a)-2 PPG Preprocessed Signals-Amplitude

0.25 PPG amplitude

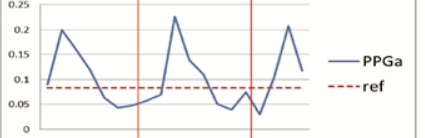

C. (b) GSR Preprocessed Signals-Mean

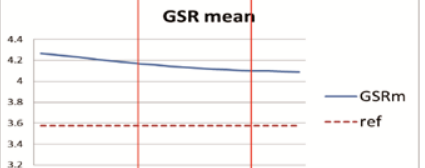

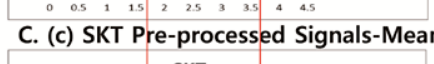

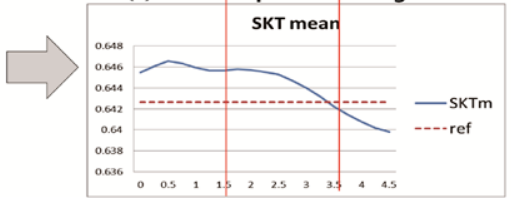

Fig. 8. A snapshot of subject A's physiological signal patterns when subject A experiences the changes of emotion picture content under "Fatigue" affective states. 


\section{Table 3}

Comparing results of the subject's affective states estimated by using physiological signals and the subjective feeling for the individual and assimilative test

\begin{tabular}{lcc}
\hline $\begin{array}{l}\text { Subjects } \\
\text { Individual }\end{array}$ & $\begin{array}{l}\text { Agreement of } \\
\text { individual emotion } \\
\text { response (\%) }\end{array}$ & $\begin{array}{l}\text { Agreement of } \\
\text { assimilative emotion } \\
\text { response }(\%)\end{array}$ \\
\hline$\# 1$ & 0.50 & 0.75 \\
$\# 2$ & 0.86 & 0.57 \\
$\# 3$ & 0.71 & 0.86 \\
$\# 4$ & 0.50 & 0.38 \\
$\# 5$ & 0.35 & 0.50 \\
$\# 6$ & 0.86 & 0.90 \\
$\# 7$ & 0.53 & 0.29 \\
$\# 8$ & 0.71 & 0.53 \\
$\# 9$ & 0.47 & 0.65 \\
$\# 10$ & 0.47 & 0.60 \\
$\# 11$ & 0.73 & 0.76 \\
$\# 12$ & 0.65 & 0.73 \\
$\# 13$ & 0.65 & 0.67 \\
$\# 14$ & 0.75 & 0.80 \\
Average & 0.62 & 0.64 \\
\hline
\end{tabular}

subject's emotional changes detected by the system had varied over time during this task. Typically, subjects showed, on average, 16 occurrences of emotional changes, ranging from 12 to 20 occurrences, within the two-minute session. The most frequently occurring emotion that matched in both the objective and subjective measurement was "Relaxing" followed by "Fatigue" and then "Pleasure". On contrast, the emotion that showed the least agreement was "Neutral".

As shown in Table 3, the agreement of individual emotion response showed that the accuracy between the self-reported subjective feeling and the subject's affective state as detected by the physiological signals was, on average, about $62 \%$, with percentages ranging from $35 \%$ (Subject \#5) to $86 \%$ (Subject \#6). The median was $65 \%$. An agreement of $62 \%$ was high enough for the intuitive subject responses and the untrained physiological responses since most subjects had never used physiological sensors before and none of them were familiar with the emotional digital photo frame system.

\subsection{Agreement of assimilative emotion response}

As shown in Table 3, the agreement of assimilative emotion response showed that the accuracy between the self-reported subjective feeling and the partner's affective state presented by the emotional pho- tograph was, on average, 64\%, with percentages ranging from 29\% (Subject \#7) to 90\% (Subject \#6). The median was $66 \%$. The rate varied greatly for each pair of friends because the experiment had been conducted without training. Ten out of fourteen subjects showed the increased accuracy on the degree of agreement of assimilative emotion responses as compared to the degree of agreement between individual emotion responses. The results indicated that most subjects were able to convey their emotion to their partner.

Table 4 shows the cross tabulation analysis results of emotion sharing between the partners. The most frequently occurring affective state of oneself was "Relaxing" followed by "Fatigue" and then "Pleasure". On the other hand, the least occurrence emotion was "Neutral". It meant that the subjects were affected one way or the other by their partner. The detailed analysis using the Chi-square test showed there to be a strong association of emotional states between the partners. The probability value $\mathrm{p}$ computed by the Chi-square test was very small $(\mathrm{p}=0.000)$, and it indicated that the degree to which the subject's emotion was affected by his or her partner's emotion to be significant. That is, emotion sharing between the partners did actually occur.

Figure 9 shows a bar graph of cross correlation percentage $(\%)$ between one and the friend's emotion. It revealed that one and the friend shared the same affective state most of the time. For example, the friend reacted $69.3 \%$ of the "Pleasure" state when given one's "Pleasure" state. Similarly, the percentage of matched affective states was $51.5 \%, 64.9 \%, 61.7 \%$ and $67.1 \%$ for "Stress", "Fatigue", "Relaxing" and "Neutral" respectively. In particular, the strongest association between one and the friend's emotion was "Pleasure". That is, the sharing of "Pleasure" was the most influential between the partners.

In the previous study, the Emotionally Intelligent Content, which dynamically changed its appearance in response to user emotion, induced more user emotional changes as compared to the original content [25]. This experiment found that the same affective states had arisen between two users from sharing this emotional photo frame system. So, it seemed that the pre-defined or agreed forms of emotional expression had benefited from establishing the common grounds between partners.

Interestingly, this emotional photo frame system adopting user-specific emotion reaction rule also helped users perceive and understand their partners' emotion intuitively. The result was in line with the 
Table 4

Friend $\times$ oneself emotion cross tabulation analysis

\begin{tabular}{|c|c|c|c|c|c|c|c|c|}
\hline & & & \multicolumn{5}{|c|}{ Oneself } & \multirow[t]{2}{*}{ Total } \\
\hline & & & Pleasure & Stress & Fatigue & Relaxing & $\overline{\text { Neutral }}$ & \\
\hline \multirow{10}{*}{ Friend } & \multirow{2}{*}{ Pleasure } & Frequency & 160 & 33 & 0 & 38 & 0 & 231 \\
\hline & & Friend (\%) & 69.3 & 14.3 & 0.0 & 16.5 & 0.0 & 100.0 \\
\hline & \multirow{2}{*}{ Stress } & Frequency & 29 & 121 & 73 & 0 & 12 & 235 \\
\hline & & Friend (\%) & 12.3 & 51.5 & 31.1 & 0.0 & 5.1 & 100.0 \\
\hline & \multirow{2}{*}{ Fatigue } & Frequency & 0 & 63 & 192 & 0 & 41 & 296 \\
\hline & & Friend (\%) & 0.0 & $21.3 \%$ & 64.9 & 0.0 & 13.9 & 100.0 \\
\hline & \multirow{2}{*}{ Relaxing } & Frequency & 113 & 10 & 42 & 318 & 32 & 515 \\
\hline & & Friend $(\%)$ & 21.9 & $1.9 \%$ & 8.2 & 61.7 & 6.2 & 100.0 \\
\hline & \multirow{2}{*}{ Neutral } & Frequency & 0 & 21 & 24 & 10 & 112 & 167 \\
\hline & & Friend $(\%)$ & 0.0 & 12.6 & 14.4 & 6.0 & 67.1 & 100.0 \\
\hline \multirow{2}{*}{ Total } & & Frequency & 302 & 248 & 331 & 366 & 197 & 1444 \\
\hline & & Friend (\%) & 20.9 & 17.0 & 22.9 & 25.3 & 13.6 & 100.0 \\
\hline
\end{tabular}

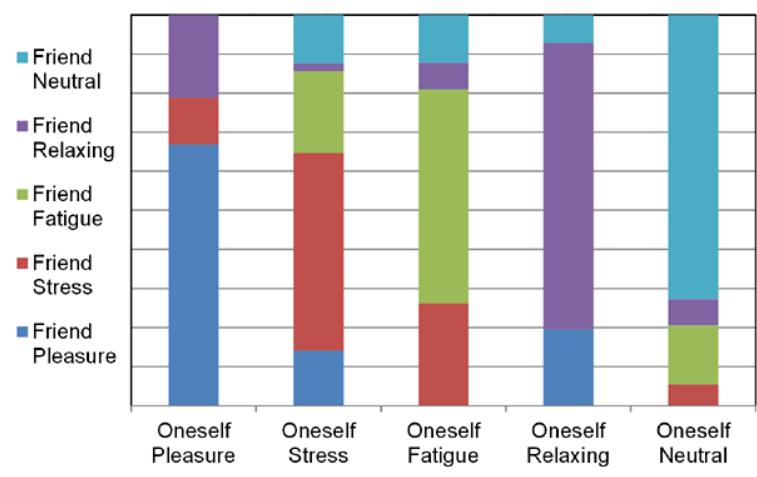

Fig. 9. A bar graph of oneself $\times$ friend emotion cross correlation percentage $(\%)$.

previous work on personalized emotional expressions to improve natural human to robot interaction [33]. It may have happened because this system supports subtle visual and aural rendering aspects used for the emotional expression on the photo.

Unfortunately, the participants recruited in this experiment were female dominant since they had to be in close relationship as the partner. It may consider that such positive results obtained in this experiment may be because of female users who spend more time with social networking service. However, the previous research investing on emotion contagious via the emotional photo also showed that the gender of the viewer had weak effects [38].

\section{Conclusions and future works}

This research explored the interactive emotional digital photo frame system to mediate individual af- fective states among close relationships such as family members or intimate friends. This proposed system captures a user's emotion non-intrusively using ANS physiological sensors. These emotional data are then shared via the server so that the user's emotion changes are automatically reflected on the remote user's digital picture frame by using user-specific emotional reaction rules. The emotional reaction rules are the specification of various colors and patterns on the photo defined by the owner of picture frame. This mechanism allows the owner of the emotion-receiving device to be aware of his or her counterpart's emotion and to be easily assimilated into the partner's affective states.

In this research, an experiment was also conducted to evaluate the system effectiveness and the feasibility of emotion awareness and sharing between users. First, the participants verified their feeling of emotion while using this emotional digital photo frame system (i.e., sensing their affective states by physiological sensors). Then, the participants verified their feeling of emotion affected by his or her partner's affective state changes displayed on the emotional photo. The results showed that the agreement of individual emotional response and the agreement of assimilative emotional response was $62 \%$ and $64 \%$ on average respectively, without training. Also, the cross tabulation analysis using the Chi-square test revealed that there was a strong association of emotional states between the partners.

Interactive emotion experience sharing improves the usage of an affective system significantly and it provides users with all the benefits of a natural interaction. In particular, this interactive emotional digital photo 
frame system demonstrated more scalable personalized emotion sharing services via the emotion share server, without the need for any procedural or coordinated systematic protocols adopted by many previous works. One of the important aspects found in this research was that emotion responses depended solely on the regulation capability of the individual because user emotion states frequently differ from one person to another and are very sensitive to day-to-day variations.

In the near future, the current prototype of this system will be enhanced to be more unobtrusive by physiological sensors embedded in the emotional digital picture frame. Hence, a user will be able to simply touch the frame for emotion detection. It also plans to improve the emotion recognition system associated with additional personal speech or face recognition algorithm to increase the accuracy without the need for any prior training. Currently the facial expression recognition algorithm is developing to extract and map feature points from the user to the avatar.

The latest smartphones in the market come with a fingerprint and heart rate sensor. In addition, the current technology trends show a lot more built-in physiological sensors in smart devices. More recently, there are growing interests on communication tools for conveying personal emotion to others since more increased numbers of people are in long-distance relationships. In this research, it was realized that a wide variety of emotional expressions could be existed even though the user affective state was the same.

This emotional photo sharing system would be more useful if family members or couples try to mediate their affective experiences with their counterparts. However, there might be some bias in the current experiment results based on the fact that subjects already have an in-depth knowledge about the likes or dislikes and emotional responses of their friends. Therefore, in the future, a controlled experiment (such as, a double blind test) will be conducted using this system for mediating affective experience between people in close relationships. Such an emotion-sharing service in response to user affective states including individual's own preferred contents provided by this system will be more popular in the near future.

\section{Acknowledgement}

This research was supported by the research fund of Dankook University in 2013.

\section{References}

[1] S.M. Ahn, M.C. Whang, D.K. Kim, J.H. Kim and S.I. Park, Real-time emotion recognition technology using individualization process emotion technology, Journal of Korea Society for Emotion and Sensibility 15(1) (2012), 133-140.

[2] A. Chang, B. Resner, B. Koerner, X.C. Wang and H. Ishii, LumiTouch: an emotional communication device, in: Proc. of CHI '01 Extended Abstracts on Human Factors in Computing Systems, ACM, New York, NY, USA, 2001, pp. 313-314.

[3] C.Y. Chang, S. Tsai, C.J. Wang and P.C. Chung, Emotion recognition with consideration of facial expression and physiological signals, in: Proc. of IEEE Symposium on Computational Intelligence in Bioinformatics and Computational Biology, IEEE Computer Society, Washington DC, USA, 2009, pp. 278-283.

[4] C.Y. Chang, J.Y. Zheng and C.J. Wang, Based on support vector regression for emotion recognition using physiological signals, in: Proc. of the International Joint Conference on $\mathrm{Neu}$ ral Networks, IEEE Computer Society, Washington DC, USA, 2010, pp. 1-7.

[5] R. Cowie, E.D. Cowie, N. Tspapatsoulis, G. Votsis, S. Kollians, W. Fellenz and J.G. Taylor, Emotion recognition in human computer interaction, IEEE Signal Processing Magazine 18(1) (2002), 32-80.

[6] Y.J. Eum, E.H. Jang, B.J. Park, S.S. Choi, S.H. Kim and J.H. Sohn, Emotion recognition by ANS responses evoked by negative emotion, in: Proc. of the International Conference on Engineering and Industries (ICEI), IEEE Computer Society, Washington DC, USA, 2011, pp. 1-4.

[7] C.A. Frantzidis, C. Bratsas, M.A. Klados, E. Konstantinidis, C.D. Lithari, A.B. Vivas, C.L. Papadelis, E. Kaldoudi, C. Pappas and P.D. Bamidis, On the classification of emotional biosignals evoked while viewing affective pictures: an integrated data-mining-based approach for healthcare applications, IEEE Transactions on Information Technology in Biomedicine 14(2) (2010), 309-318.

[8] G. Gay, J.P. Pollak, P. Adams and J.P. Leonard, Pilot study of Aurora, a social, mobile-phone-based emotion sharing and recording system, Journal of Diabetes Science and Technology 5(2) (2011), 325-332.

[9] E.H. Jang, B.J. Park, S.H. Kim, Y. Eum and J. Sohn, Identification of the optimal emotion recognition algorithm using physiological signals, in: Proc. of International Conference on Engineering and Industries (ICEI), IEEE Computer Society, Washington DC, USA, 2011, pp. 1-6.

[10] S. Jerritta, M. Murugappan, R. Nagarajan and K. Wan, Physiological signals based human emotion recognition: a review, in: Proc. of the 7th IEEE International Colloquium on Signal Processing and Its Applications (CSPA), IEEE Computer Society, Washington DC, USA, 2011, pp. 410-415.

[11] R. Kikin-Gil, BuddyBeads: techno-jewelry for non verbal communication within groups of teenage girls group, Journal of Personal and Ubiquitous Computing 10(2-3) (2006), 106109.

[12] D.K. Kim, J.H. Kim, E.C. Lee, M.C. Whang and Y. Cho, Interactive emotional content communications system using portable wireless biofeedback device, IEEE Transactions on Consumer Electronics 57(4) (2011), 1929-1936.

[13] D.K. Kim, S.M. Ahn, S.M. Park and M.C. Whang, Interactive emotional lighting system using physiological signals, IEEE 
Transactions on Consumer Electronics 59(4) (2013), 765771.

[14] J. Kim and E. André, Emotion recognition based on physiological changes in music listening, IEEE Transactions on Pattern Analysis and Machine Intelligence 30(12) (2008), 20672083.

[15] J.H. Kim, M.C. Whang, J.Y. Kim and J.C. Woo, The study on emotion recognition by time dependent parameters of autonomic nervous response, Korean Journal of Korean Society for Emotion \& Sensibility 11(4) (2008), 637-644.

[16] M. Kim, K.S. Park, D.K. Kim and Y. Cho, Emotional intelligent contents: Expressing user's own emotion with contents, in: Entertainment Computing, Lecture Notes in Computer Science, Vol. 6972, 2011, pp. 391-394.

[17] S.D. Kreibig, Autonomic nervous system activity in emotion: a review, Biology Psychology 84(3) (2010), 394421.

[18] R.J. Larsen and E. Diener, Promises and problems with the circumplex model of emotion, Review of Personality and Social Psychology 13 (1992), 25-59.

[19] O. Liechti and T. Ichikawa, A digital photography framework supporting social interaction and affective awareness, in: Handheld and Ubiquitous Computing, Lecture Notes in Computer Science, Vol. 1707, 1999, pp. 186-192.

[20] A. Neviarouskaya, H. Prendinger and M. Ishsizuka, EmoHeart: conveying emotions in second life based on affect sensing from text, Advanced in Human-Computer Interaction 2010 (2010), 77-84.

[21] A. Neyem, C. Aracena, C.A. Collazos and R. Alarcon, Designing emotional awareness devices: what one sees is what one feels, Ingeniare. Revista Chilena de Ingenieria 15(3) (2007), 227-235.

[22] T.L. Nguyen, Emuplayer: music recommendation system based on user emotion using vital-sensor, Bachelor's thesis, Keio University, Japan, 2010

[23] C. Park, D. Looney and D.P. Mandic, Estimating human response to taste using EEG, in: Proc. of the Annual International Conference of the IEEE Engineering in Medicine and Biology Society, IEEE Computer Society, Washington DC, USA 2011, pp. 6331-6334.

[24] J.S. Park, J.H. Kim and Y.H. Oh, Feature vector classification based speech emotion recognition for service robots, IEEE Transactions on Consumer Electronics 55(3) (2009), 15901596.

[25] K.S. Park, Y. Cho and D.K. Kim, A framework for the creating, expressing and sharing of user's emotion, International Journal of Multimedia and Ubiquitous Engineering 9(1) (2014), 425-442, 1.
[26] P.C. Petrantonakis and L.J. Hadjileontiadis, A novel emotion elicitation index using frontal brain asymmetry for enhanced EEG-based emotion recognition, IEEE Transactions on Information Technology in Biomedicine 15(5) (2011), 737-746.

[27] A.F. Rovers and H.A. van Essen, HIM: a framework for haptic instant messaging, in: Proc. of CHI '04 Extended Abstracts on Human Factors in Computing Systems, ACM, New York, NY, USA, 2004, pp. 1313-1316.

[28] J.A. Russell, A circumplex model of affect, Journal of Personality and Social Psychology 39(6) (1980), 1161-1178.

[29] B. Schneiderman, Designing the User Interfaces: Strategies for Effective Human-Computer Interaction, 3rd edn, AddisonWesley Longman Publishing Co., Inc., Boston, MA, USA, 1998.

[30] M. Soleymani, M. Pantic and T. Pun, Multimodal emotion recognition in response to videos, IEEE Transactions on Affective Computing 3(2) (2012), 211-223.

[31] J.F. Thayer and G.J. Siegle, Neurovisceral integration in cardiac and emotional regulation, IEEE Engineering in Medicine and Biology Magazine 21(4) (2002), 24-29, 4.

[32] E.L. Van den Broek, Ubiquitous emotion-aware computing, Personal Ubiquitous Computing 17(1) (2013), 53-67.

[33] M. Vircikova, P. Sincak and D.H. Kim, Personalized emotional expressions to improve natural human-humanoid interaction, Robot Intelligence Technology \& Applications, Advances in Intelligent Systems and Computing 208 (2013), 691-702.

[34] D. Vronay, S. Farnham and J. Davis, PhotoStory: pre-serving emotion in digital photo sharing, Microsoft Research Internal Report, 2001.

[35] R. Yamanishi, Y. Ito and S. Kato, Automated song selection system complying with emotional requests, in: Entertainment Computing, Lecture Notes in Computer Science, Vol. 6972, 2011, pp. 367-370.

[36] Y. Wang and L. Guan, Recognizing human emotional state from audiovisual signals, IEEE Transactions on Multimedia 10(5) (2008), 659-668.

[37] M.C. Whang, J.S. Lim, K.R. Park, Y.J. Cho and W. Boucsein, Are computers capable of understanding our emotional states? in: Engineering Psychology and Ergonomics, Lecture Notes in Computer Science, Vol. 4562, 2007, pp. 204-211.

[38] B. Wild, M. Erb and M. Bartels, Are emotions contagious? Evoked emotions while viewing emotionally expressive faces: quality, quantity, time course and gender difference, Psychiatry Research 102(2) (2001), 109-124.

[39] Z. Zeng, J. Tu, B.M. Pianfetti and T.S. Huang, Audio-Visual affective expression recognition through multistream fused HMM, IEEE Transactions on Multimedia 10(4) (2008), 570577. 